\title{
A COVID-19 e o impacto da pandemia na saúde mental dos profissionais de
}

\section{fisioterapia: uma revisão integrativa}

\author{
COVID-19 and the impact of the pandemic on the mental health of physical therapy professionals:
} an integrative review

COVID-19 y el impacto de la pandemia en la slaud mental de los profesionales de fisicoterapia: una revisión integrativa

\author{
Maria de Nazaré Silva \\ ORCID:https://orcid.org/0000-0002-9541-3152 \\ Centro Universitário Santo Agostinho, Brasil \\ E-mail: naza.nat@hotmail.com \\ Liana Dantas Da Costa e Silva Barbosa \\ ORCID: https://orcid.org/0000-0002-8191-102X \\ Centro Universitário Santo Agostinho, Brasil \\ E-mail: dantasliana@bol.com.br
}

\begin{abstract}
Resumo
Em meio a uma pandemia que foi detectada no mundo no final de 2019, como um surto global de histórico desconhecido em que é caracterizado pelo quadro comum de fadiga, tosse, febre alta e principalmente dificuldade respiratória a todos os pacientes, e diagnosticada atualmente como COVID-19, tendo como causador o SARS-CoV-2. O presente estudo trata-se de uma revisão integrativa, na qual a amostra de 27 artigos foi composta a partir de um levantamento de dados através da busca na BDENF onde estão indexadas as bases de dados eletrônicas utilizadas na pesquisa: SCIELO (Scientific Eletronic Library Online), LILACS (Literatura Latino-Americana, nos anos de 2020 a 2021. Os descritores foram cruzados entre si utilizando o marcadore booleano AND . A coleta foi realizada no período de Agosto a Novembro de 2021. Devido a alta transmissibilidade do SARS-CoV-2 a quarentena e o isolamento social têm sido utilizados como principal estratégia de contenção do vírus, desencadeando um aumento nos casos de depressão, ansiedade e estresse na população geral, incluindo os profissionais da saúde. A importância da implementação de estratégias e ou intervenções para que a população tenha acesso à medidas de apoio emocional diante a pandemia do SARS-CoV-2, seja presencial ou online. Levando em consideração as diferentes populações afetadas, recomenda-se lidar com as consequências na saúde mental diante da pandemia e intervenções psicológicas em resposta às necessidades individuais no decorrer do surto pandêmico.
\end{abstract}

Palavras-chave: Fisioterapia; Infecções por coronavírus; Saúde mental.

\begin{abstract}
Amid a pandemic that was detected in the world at the end of 2019, as a global outbreak of unknown history in which it is approved by the common picture of fatigue, cough, high fever and especially respiratory distress in all patients, and currently diagnosed as Coronavirus 2019 (COVID-19). The guiding question: What are the impacts on the mental health of physical therapy professionals during a COVID-19 pandemic? Therefore, the general objective that guided the research was: To analyze the scientific evidence on the impact on the psychological of physical therapy professionals during the COVID-19 pandemic. This is a systematic review, which is a review method that performs many functions criticisms; they can provide summaries of the state of knowledge in a field, from which future research priorities can be identified; in addition to identifying problems in primary research to be corrected in future studies; and generate or evaluate theories about how or why phenomena occur.
\end{abstract}

Keywords: Physiotherapy; Coronavirus; Mental health.

\section{Resumen}

En medio de una pandemia que se detectó en el mundo a finales de 2019, como un brote mundial de historia desconocida en el que se aprueba por el cuadro común de fatiga, tos, fiebre alta y sobre todo dificultad respiratoria en todos los pacientes, y actualmente diagnosticado como Coronavirus 2019 (COVID-19). Al elegir su área de investigación, los motivos del investigador fueron, además de la búsqueda de conocimiento, la gran cantidad de casos de infectados por el nuevo virus Corona y los impactos en la salud mental de los profesionales de la salud. La pregunta guía: ¿Cuáles son los impactos en la salud mental de los profesionales de fisioterapia durante una pandemia de COVID-19? Por tanto, el objetivo general que orientó la investigación fue: Analizar la evidencia científica sobre el impacto psicológico de los profesionales de la fisioterapia durante la pandemia de COVID-19. Se trata de una revisión 
sistemática, que es un método de revisión que cumple muchas funciones críticas; pueden proporcionar resúmenes del estado del conocimiento en un campo, a partir de los cuales se pueden identificar las futuras prioridades de investigación; además de identificar problemas en la investigación primaria a corregir en estudios futuros; y generar o evaluar teorías sobre cómo o por qué ocurren los fenómenos.

Palabras clave: Fisioterapia; Coronavirus; Salud mental.

\section{Introdução}

Em meio uma pandemia que foi detectada no mundo no final de 2019, como um surto global de histórico desconhecido em que é caracterizado pelo quadro comum de fadiga, tosse, febre alta e principalmente dificuldade respiratória a todos os pacientes, e diagnosticada atualmente como Coronavírus 2019 (COVID-19) (Sexton et al., 2018).

Portanto, é preciso frisar que, assim como os pacientes, esses profissionais também possuem pessoas que os amam, para as quais precisam voltar com saúde física e mental. Diante desse novo cenário global, a COVID-19 trouxe novos desafios e mudança no que diz respeito à saúde mental dos profissionais da área da saúde.

Os profissionais da saúde diariamente enfrentam condições de trabalho instáveis, em um ambiente marcado pela falta de segurança, infraestrutura inadequada e pelos riscos nele presentes. Isto influi em níveis altos de desgaste profissional, adoecimento físico e psicológico, má qualidade de vida e assistência à saúde (Rego, 2020).

Essas situações são mais comuns aos profissionais atuantes na linha de frente de combate a COVID-19, devido às longas horas de trabalho, preocupação com o estado de saúde dos pacientes, sobretudo ao que diz respeito a respiração, e com a escassez de Equipamentos de Proteção Individual (EPI), principalmente em países com recursos limitados.

A importância da pesquisa é evidenciada no momento em que a doença revela-se como algo novo e incerto no cenário mundial e servirá como fonte de informação à sociedade em geral. O pesquisador ao escolher sua área de pesquisa teve como motivos, além da busca por conhecimento, o grande número de casos de infectados pelo novo Corona vírus e os impactos na saúde mental nos profissionais da área da saúde.

A questão norteadora: Quais os impactos na saúde mental dos profissionais de fisioterapia durante a pandemia do COVID-19?

Sendo assim o objetivo geral que norteou a pesquisa foi: Analisar as evidências científicas sobre as o impacto no psicológico dos profissionais de fisioterapia durante a pandemia do COVID 19.

Trata-se de uma revisão sistemática, que é um método de revisão que desempenha muitas funções críticas; podem fornecer sínteses do estado do conhecimento em um campo, a partir do qual as futuras prioridades de pesquisa podem ser identificadas; além de identificar problemas na pesquisa primária para serem corrigidos em estudos futuros; e gerar ou avaliar teorias sobre como ou por que os fenômenos ocorrem.

Para a seleção dos artigos a serem utilizados nessa pesquisa adotou-se como critérios de inclusão: artigos primários de abordagem qualitativa que tenham como participantes os profissionais de fisioterapia que estão na linha de frente do Covid-19, e que responda a com publicação na íntegra, publicados entre 2019 a 2021, nos idiomas: português, inglês e espanhol.

\section{A pandemia e Seus Impactos Psicológicos}

\subsection{CORONA VÍRUS: aspectos conceituais, diagnóstico e consequências}

Foi definida pela Organização Mundial da Saúde (OMS) como uma pandemia em março de 2020 onde foram fechadas escolas, restaurantes, estabelecimentos públicos e privados, pois comprovou-se que os pacientes podem ter contraído o vírus da síndrome respiratória aguda grave (SARS-CoV-2) e apresentar um estado assintomático, potencializando a transmissibilidade do vírus que costuma ocorrer pelo ar ou por contato pessoal com secreções contaminadas através das gotículas de saliva, espirro, tosse, contato pessoal próximo, como aperto de mão ou toque e dentre outros. 
A principal forma de transmissão do coronavírus está pelo contato de fluídos corporais (direta) e/ou por meio das superfícies dos objetos (indireta). Com isso, os profissionais da saúde tornaram-se os agentes de frente dessa situação ao combate a COVID-19. Nesse sentido, urge a atuação do Cirurgião-Dentista (CD) como profissional da saúde que representa um risco elevado a transmissão desse vírus.

Diante desse novo cenário global, a COVID-19 trouxe novos desafios e mudança no aspecto da biossegurança nos consultórios odontológicos. O Coronavírus é um tipo de vírus que pode ser transmitido de animais para humanos; em tais casos, esse vírus sofre mutação quando passa para os humanos, levando ainda mais à disseminação entre humanos (Chan Et Al., 2020b).

Um Coronavírus pode progredir em diferentes estágios, como leve, moderado e grave, e esses vírus estão no mesmo grupo da Síndrome Respiratória Aguda Grave (SARS) de 2002 e da Síndrome Respiratória do Oriente Médio (MERS) de 2012 (Huang et al., 2020).

O COVID-19 é detectado na saliva de pacientes infectados, portanto, os profissionais de fisioterapia bucal / odontológico e outros profissionais de fisioterapia em particular devem ter muito cuidado na proteção contra a propagação da doença (Sabino-Silva, Jardim \& Siqueira, 2020; To et al., 2020). A transmissão é semelhante a outras doenças respiratórias; pode ocorrer com gotículas ejetadas durante a fala, tosse ou espirro (atividades do aparelho respiratório) e também por aerossóis empregados durante procedimentos clínicos (Sabino-Silva, Jardim \& Siqueira, 2020).

Foi definida pela Organização Mundial da Saúde (OMS) como uma pandemia em março de 2020 onde foram fechadas escolas, restaurantes, estabelecimentos públicos e privados, pois se comprovou que os pacientes podem ter contraído o vírus da síndrome respiratória aguda grave (SARS-CoV-2) e apresentar um estado assintomático, potencializando a transmissibilidade do vírus que costuma ocorrer pelo ar ou por contato pessoal com secreções contaminadas através das gotículas de saliva, espirro, tosse, contato pessoal próximo, como aperto de mão ou toque e dentre outros (Franco, 2020).

Para profissionais de fisioterapia diretamente ligados ao atendimento de casos de COVID-19, existem alguns fatores estressores além dos que já ocorrem nos serviços de fisioterapia em geral. Cuidar de pacientes que sofrem de COVID-19 pode ter um efeito emocional importante. É comum se sentir sobrecarregado e sob pressão, mas é importante lembrar que o estresse deste momento não significa fraqueza ou incompetência profissional (Rego, 2020).

Os altos níveis de estresse apresentam uma séria ameaça à saúde mental dos profissionais, elevando as taxas de ansiedade, depressão, transtorno de estresse pós-traumático (TEPT) e comportamentos sociais negativos o que pode implicar na eficácia da jornada de trabalho dos profissionais da saúde (Sexton et al., 2018).

A doença é causada por uma das cepas que constituem a uma família do vírus, que podem causar doenças envolvendo o trato respiratório, conhecidas como SARS e MERS, denominadas respectivamente como Síndrome Respiratória Aguda Grave e Síndrome Respiratória do Oriente Médio (Odeh et al., 2020). Os sinais e sintomas principais envolvem febre, tosse seca, mialgia, apatia, perda parcial ou total do olfato e alteração ou perda total do paladar. Além de insuficiência renal e doenças respiratórias com necessidade de ventilação mecânica. (Franco et al., 2020).

As vias de transmissão da COVID-19 compreendem a transmissão direta seja por meio de tosse, espirro e perdigotos ou ainda por contato com mucosa oral, nasal e ocular. Além disso, constatou-se que os vírus podem ser transmitidos de pessoa para pessoa através do contato direto ou indireto, de saliva e fluídos (Tunãs et al., 2020).

O período de incubação tem uma média de 5 a 6 dias, porém autores afirmam que poderá se prolongar por até 14 dias, este por sua vez está sendo levado em consideração para pessoas que estão com suspeita, em observação médica ou de quarentena pela exposição de pessoas infectadas. (Meng et al., 2020)

É evidente que pneumonias associadas à ventilação mecânica podem afetar negativamente a recuperação do paciente. Portanto, essas complicações podem ser evitadas na presença de um prévio atendimento odontológico em pacientes internados 
em unidade de terapia intensiva- UTI, visando minimizar danos maiores no organismo como infecções generalizadas (Franco et al., 2020).

\subsection{Corona vírus e a saúde mental}

Durante muitos anos, a base do tratamento psiquiátrico nos países ocidentais foi baseada na internação por tempo indeterminado em manicômios Entretanto este o modelo manicomial não se mostrou eficiente, por ser cronificante e desumano. Houve fortes críticas a tal modelo, de forma específica na Europa, que eclodiram, a partir da década de 1950, por conta de um movimento político-social de desinstitucionalização psiquiátrica. O propósito deste movimento era alcançar a humanização do atendimento para com os pacientes e defender os direitos deles (Vidal., 2015).

Os integrantes alegavam que a internação prolongada não trazia auxílio na melhoria dos transtornos mentais, pelo contrário leva a cronificação da doença. E também por outro lado, com o isolamento era mais difícil que houvesse a reintegração familiar do indivíduo. (Saraceno, 2015).

A política de nacional de saúde no Brasil ao ter como foco principal a desmanicomialização, a estruturação da rede de serviços de saúde mental e o reconhecimento dos direitos de cidadania das pessoas que possuem algum transtorno mental requer ordenações nos modos de ter o cuidado e escalonar os serviços em confronto com as concepções e estratégias usuais, o que implica na definição de novos perfis dos profissionaisque atendem tais demandas (Mangia; Muramoto, 2016).

Assim, acredita-se que o papel do fisioterapeuta deve dar acessibilidade a confecção de um pensamento crítico sobre as políticas de saúde mental e a organização da rede de assistencial brasileira, como também a propagação de novas expectativas de se fazer a clínica a partir de uma nova visão, prontamente madura, das teorias e técnicas que embasam o papel do psicólogo em nosso País (Saraceno, 2001).

A oficialização da reforma era sempre adiada, por isso o próprio Ministério da Saúde passou a criar regulamentações através de portarias exigindo a assistência de uma equipe multiprofissional, acomodações devidas, espaços para laser e terapias integrantes para o tratamento dos pacientes institucionalizados. Passou também a medir as internações, taxando seu tempo máximo em 60 dias (Ribeiro., 2015).

Definiu, ainda, que os Núcleos de Atenção Psicossocial e os ambulatórios de fisioterapia ofertassem assistência familiar e comunitária, aspirando à (re) inserção social das pessoas com transtornos mentais (Brasil, 2016).

Em 6 de abril de 2001 foi finalmente decretada a Lei Federal 10.216, também conhecida como Lei Paulo Delgado. Lei esta que oficializou o atendimento psiquiátrico comunitário no Brasil, organizou sobre o tratamento mais civilizado ou humanizado, ao acolhimento às pessoas com transtornos psiquiátricos, a inclinação pelos atendimentos comunitários sobre a internação, a implementação em todo o estado territorial de serviços suplentes, as bases de funcionamento de tais serviços e o normativo das internações compulsórias (Bock, 2015).

Além dos CAPS, imaginou-se se predizia a implantação de inúmeros outras instituições com a finalidade de receber tais paciente e lhes assistencializar com um serviço mais humanizado, visando de fato o alívio do sofrimento psíquico vivido por estes, dentre estes os Centros de Atenção Psicossocial.

Há então um aspecto positivo em a loucura se tornar uma doença. Pois assim então há condições da loucura ser finalmente tratada, até então a loucura não podia ser tratada. Então ela se torna o objeto de estudo da terapêutica médica. Na época o tratamento instituído para a loucura era o tratamento moral, onde consiste no fato de que a Instituição que acolhe os loucos se reestrutura para recebê-los de forma organizada, já é visto neste momento como um tratamento terapêutico. Não se fala neste contexto em uma terapia constituída de administração de remédios, ou de práticas terapêuticas. (Gladys Swain, 2016).

No âmbito da atenção básica, muitas vezes o profissional fisioterapeuta bem como a equipe multiprofissional são os responsáveis pela efetivação dessa política. Nesse contexto, são localizadas algumas dificuldades que podem ser apontadas 
para a realização de tais ações por esses psicólogos, como a concepção de fisioterapia que norteia essas atividades. Uma concepção de fisioterapia que a toma apenas como ausência de doenças pode levar a ações pontuais, visando evitar doenças por meio de mudanças de hábitos individuais. Quando a concepção de fisioterapia excede o limite biomédico, abre-se espaço para práticas voltadas para o coletivo, comprometidas com a transformação do status quo e com a produção de sujeitos autônomos e solidários (Carvalho, 2017).

A importância da saúde mental é reconhecida pela Organização Mundial de saúde (OMS) e está refletida na definição de fisioterapia como sendo não apenas a ausência de doença ou enfermidade, mas como "um estado de completo bem-estar físico, mental e social" (OMS, 2016).

Nos últimos anos, essa definição ganhou um foco mais nítido em virtude de muitos e enormes progressos nas ciências biológicas e comportamentais. Falar de prevenção em saúde mental é algo bastante complexo, pois atualmente, mesmo com o avanço das neurociências, não é possível a prevenção de muitos dos transtornos psiquiátricos. Entretanto, esforços de pesquisa no campo da epidemiologia psiquiátrica concentram-se no aperfeiçoamento de meios de detectar pessoas em risco de desenvolvimento de transtornos mentais, como a esquizofrenia, em etapas muito precoces ou mesmo antes de sua manifestação inicial. Neste sentido, a detecção precoce dos transtornos mentais aumentaria a probabilidade de intervenção precoce, diminuindo positivamente o risco de uma evolução crônica ou de sequelas residuais graves.

De acordo com a OMS (2016), a prevenção e o tratamento adequados de certos transtornos mentais e comportamentais, por exemplo, podem reduzir os índices de suicídio, sejam essas intervenções orientadas para indivíduos, famílias, escolas ou outros setores da comunidade em geral. O reconhecimento e o tratamento precoce de depressão, dependência do álcool e esquizofrenia, por exemplo, são estratégias importantes, principalmente na prevenção do suicídio.

\subsection{A saúde mental e os profissionais de fisioterapia}

A política de saúde mental no Brasil ao ter como foco principal a desmanicomialização, a estruturação da rede de serviços de saúde mental e o reconhecimento dos direitos de cidadania das pessoas que possuem algum transtorno mental requer ordenações nos modos de ter o cuidado e escalonar os serviços em confronto com as concepções e estratégias usuais, o que implica na definição de novos perfis dos profissionaisque atendem tais demandas (Milagres, 2013).

A reforma psiquiátrica caracteriza-se como um movimento que inclui tanto a reestruturação da rede assistencial em saúde mental no Brasil, como um reposicionamento em relação ao modelo epistemológico constituinte da psiquiatria, e a reformulação do conceito de clínica de tecnologias de cuidados adotadas pelos profissionais (Amarante, 2013).

Esta nova forma de pensar, agregou para uma transição de o modelo assistencial asilar para o modelo denominado psicossocial, que tem como principais fundamentos a percepção de indivíduo elucidado na relação sujeito-sujeito, a todas as facetas de determinação do processo de adoecimento, tratamentos diversos e multidisciplinares, e a perspectiva do cuidado em rede, com ênfase no papel dos Centros de Atenção Psicossocial (CAPS) como dispositivos centrais na articulação da rede de saúde mental (Costa-Rosa, 2010).

Destacam-se as ideias de Amarante (2013), quando ele diz que a reforma psiquiátrica é, mais do que uma reestruturação na rede de assistência, é uma nova forma de posicionar o hitórico epistemológico na concepção do processo de doença - saúde e nos avanços das tecnologias de cuidado.

Assim, acredita-se que o papel psicólogo deve da acessibilidade a confecção de um pensamento crítico sobre as políticas de saúde mental e a organização da rede de assistencial brasileira, como também a propagação de novas expectativas de se fazer a clínica a partir de uma nova visão, prontamente madura, das teorias e técnicas que embasam o papel do psicólogo em nosso País (Ribeiro et al., 2010). 
A segurança dos direitos das pessoas com transtornos mentais de acordo com os estudos e pesquisas de Goulart e Durães (2010) embasa que precisa ser ininterruptamente examinada por um processo crucial de reforma psiquiátrica, seus rendimentos e seus danos, uma vez que a reforma psiquiátrica, além disso, é um procedimento barulhento.

A oficialização da reforma era sempre adiada, por isso o próprio Ministério da Saúde passou a criar regulamentações através de portarias exigindo a assistência de uma equipe multiprofissional, acomodações devidas, espaços para laser e terapias integrantes para o tratamento dos pacientes institucionalizados. Passou também a medir as internações, taxando seu tempo máximo em 60 dias (Ribeiro; Martins; Oliveira, 2010).

Definiu, ainda, que os Núcleos de Atenção Psicossocial e os ambulatórios de saúde mental ofertassem assistência familiar e comunitária, aspirando à (re) inserção social das pessoas com transtornos mentais (Brasil, 2016).

Em 6 de abril de 2001 foi finalmente decretada a Lei Federal 10.216, também conhecida como Lei Paulo Delgado. Lei esta que oficializou o atendimento psiquiátrico comunitário no Brasil, organizou sobre o tratamento mais civilizado ou humanizado, ao acolhimento às pessoas com transtornos psiquiátricos, a inclinação pelos atendimentos comunitários sobre a internação, a implementação em todo o estado territorial de serviços suplentes, as bases de funcionamento de tais serviços e o normativo das internações compulsórias (Mota, 2016).

Além dos CAPS, imaginou-se se predizia a implantação de inúmeros outras instituições com a finalidade de receber tais paciente e lhes assistencializar com um serviço mais humanizado, visando de fato o alívio do sofrimento psíquico vivido por estes, dentre estes as residências terapêuticas. (Brasil, 2014).

De acordo com o exposto, os Serviços Residenciais Terapêuticos (SRT), observam como uma intensa e imprescindível deliberação de precaução à saúde mental, bem como uma forma de tática restauradora do sistema de físioterapia eficaz hoje em dia. (Brasil, 2014).

Os SRT são casas que estão inseridas em bairros urbanas, que são destinadas a cuidar dos portadores de sofrimento psíquico, tendo como objetivo a progressão de inclusão social de todos os moradores, respeitando sua individualidade e contando com: suporte de infraestrutura física e equipe multidisciplinar que tenha por objetivo acompanhar os moradores e ajuda-los a recuperar ou a construir autonomia e independência ajustada as necessidades deles; e também projetos de terapia individualizados. (PTI) (Brasil, 2014a).

Ao que diz respeito ao suporte, é preciso que elas sejam assistidas ou coordenadas de acordo com o grau de dependência ou independência que os moradores tenham. Nas RT que tenham uma quantidade com pessoas menos independentes, podem ser dados cuidados profissionais, da mesma forma que, nas residências com pessoas mais autônomas, algumas atividades terapêuticas podem ser feitas de acordo com os recursos sanitários que atendem território (Mota, 2016).

Em qualquer uma das duas circunstâncias citadas é importante ter como "norma" que cumpre às equipes de fisioterapia da família orientar todas as residências que possuem no território, com a supervisão e o apoio essencial dos outros serviços de atenção psicossocial.

Sabe-se que é na convivência do dia-dia que se instaura uma nova forma de tratar e acolher o sofrimento psíquico, baseado na escuta de cada demanda de acordo com sua especificidade, foi elaborado um projeto terapêutico individual (PTI), sendo administrado como aliança de sustentação: cuidado particularizado; atuações focalizadas nas demandas singulares de cada residente e estímulo ao local em que há relação de confiança com todos os profissionais e com a sugestão do sistema (Milagres, 2013). 


\section{Metodologia}

Trata-se de uma revisão integrativa de literatura. Para atingir o objetivo foi definida a questão norteadora da pesquisa: Quais as evidências científicas acerca do impacto da pandemia do SARS-CoV-2 na saúde mental de profissionais de fisioterapia?

Trata-se de uma pesquisa bibliográfica de revisão integrativa estruturada em seis etapas distintas: elaboração da questão de pesquisa; busca na literatura dos estudos primários; extração de dados dos estudos primários; avaliação dos estudos primários que compõem a revisão; análise e síntese dos resultados da revisão; apresentação da revisão integrativa (Camargo et al. 2017).

Para constituição da amostra foi realizado um levantamento bibliográfico, no período de agosto a novembro de 2020, através de busca eletrônica, onde estão indexadas as bases de dados eletrônicas utilizadas nesta pesquisa: BDENF, SCIELO (Scientific Eletronic Library Online), LILACS (Literatura Latino-Americana e do Caribe em Ciências da Saúde), nos anos de 2020 a 2021. Foram utilizados os descritores: Infecções por Coronavírus (Coronavírus Infections), Pandemias (Pandemics), Saúde mental (Mental Health) e Fisioterapia (Physiotherapy) contidos no site dos Descritores em Ciências da Saúde (DeCS) e as palavras-chave: Fisioterapia, Infecções por Coronavírus e Saúde Mental. Esses descritores foram cruzados entre si utilizando o marcador booleano AND.

Após os cruzamentos já mencionados foram adotados como critérios de inclusão: artigos originais, artigos online disponíveis na íntegra, publicados no período de 2020 e 2021, nos idiomas Português e Inglês que seguem os descritores acima, que abordavam os impactos da pandemia do SARS-CoV-2 na saúde mental de profissionais de fisioterapia. Foram excluídos da amostra: artigos em forma de estudos de caso, ensino de apostilas, cartas e editoriais, artigos com duplicidade ou que não estivessem alinhados aos descritores e objetivos.

Trata-se de uma revisão integrativa, na qual a seleção das publicações para a revisão seguiu as orientações da estratégia de busca PICO (Quadro 1), onde a questão de pesquisa estabelecida conta com 4 componentes: P (Paciente), I (Intervenção), C (Comparação/controle) e O (Desfecho).

Quadro 1. Descrição da estratégia PICO. Teresina, Piauí, Brasil, 2021.

\begin{tabular}{|c|c|}
\hline ACRÔNIMO & ANÁLISE \\
\hline $\begin{array}{c}\text { P } \\
\text { (Paciente) }\end{array}$ & Profissionais de fisioterapia \\
\hline $\begin{array}{c}\text { I } \\
\text { (Intervenção ou indicado) }\end{array}$ & $\begin{array}{c}\text { Impactos na saúde mental dos profissionais de fisioterapia sofridos na } \\
\text { pandemia do SARS COVID-19 }\end{array}$ \\
\hline $\begin{array}{c}\mathrm{C} \\
\text { (Comparação ou controle) }\end{array}$ & Enfrentamento dos impactos na saúde mental. \\
\hline $\begin{array}{c}\text { O } \\
\text { (Outcomes /Desfecho) }\end{array}$ & E \\
\hline
\end{tabular}

Fonte: Autores (2021).

Os artigos escolhidos foram lidos, cautelosamente, para identificar quais informações seriam extraídas para o presente estudo. A coleta dos dados foi auxiliada por um formulário de categorização de artigos que permitiu armazenar dados referentes aos aspectos metodológicos e temáticos das produções incluindo as seguintes informações: título, ano de publicação, autores, periódico de publicação e resultados das pesquisas. Após avaliação crítica dos autores, seguiu-se a interpretação e apresentação de uma síntese dos resultados encontrados.

Ao final do processo de seleção foi encontrado um total de 710 artigos relacionados com a temática, deste total foram identificadas 250 produções no LILACS, na SCIELO e 230 na BDENF. 
Após a utilização dos filtros e a leitura dos resumos e resultados foram excluídos 330 artigos e selecionados artigos completos, restando 380 artigos. Logo após a leitura dos resumos e excluindo aqueles que não atenderam aos critérios de inclusão, foram selecionados os textos completos de 20 artigos, a partir desses artigos selecionados 15 artigos foram incluídos no estudo encontrados a partir das citações; foram escritos em português, inglês e espanhol, sendo designadas 10 publicações que atenderam aos critérios de inclusão e exclusão previamente instituídos (Figura 1).

$\mathrm{Na}$ seleção das publicações foram consideradas as recomendações do Preferred Reporting Items for Systematic Reviews and Meta-Analyses (PRISMA): dois pesquisadores independentes realizaram a seleção das publicações identificadas, avaliando criteriosamente a metodologia dos artigos considerados relevantes para o tema em estudo; a seleção ocorreu por meio dos dois pesquisadores independentes obedecendo aos critérios de inclusão e exclusão previamente definidos.

Figura 1. Processo de seleção dos estudos. Teresina, 2021.

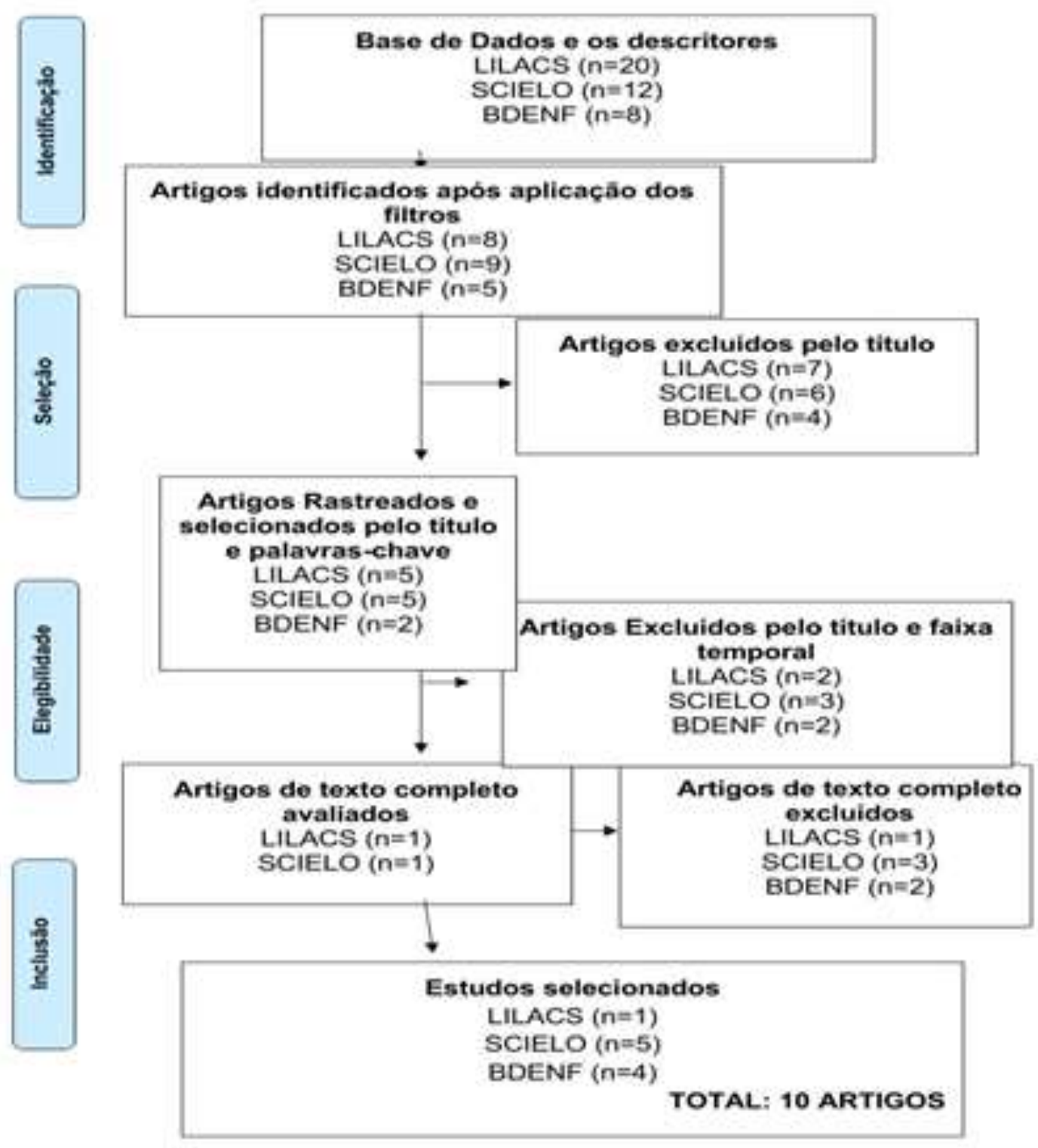

Fonte: Autores (2021).

Os 10 artigos selecionados foram lidos para determinar quais informações seriam extraídas e utilizadas no estudo. Estes artigos foram caracterizados com o auxílio de um formulário estruturado de artigos para armazenar dados referentes aos 
aspectos metodológicos e temáticos dos estudos, incluindo as seguintes informações: autores, ano e local de publicação, título, periódico de publicação e resultados da pesquisa.

A análise de dados ocorreu pela leitura detalhada dos estudos, permitindo a retirada das ideias mais relevantes de cada autor, considerando os resultados evidenciados e as conclusões de cada publicação. Para gerir o processo de análise, três fases do processo analítico de Minayo (2017) foram utilizadas: pré-análise (etapa de leitura do conteúdo para identificar as principais ideias dos artigos escolhidos), exploração do material (fase de construção de grupos temáticos mediante escolha dos conteúdos de cada pesquisa selecionada) e agrupamento dos resultados obtidos (interpretação dos resultados e comparação com a literatura existente).

Após coleta de dados e caracterização dos estudos selecionados, as informações foram organizadas e apresentadas em um quadro onde os conteúdos descritivos das principais evidências e conclusões foram organizados em agrupamentos temáticos pela semelhança das ideias dos autores.

Os resultados e as evidências das publicações foram discutidos apartir de teóricos que se relacionam com a temática do estudo e apresentadas na discussão desta pesquisa. A presente revisão foi estruturada como artigo, seguindo-se as normas legais dispostas pela American Psychological Association (APA), estabelecendo a reprodução das evidências consideráveis contidas nos resultados dos artigos analisados conforme descritos, tendo em vista, a autenticidade das principais ideias apresentadas pelos seus autores, em citações diretas ou parafraseadas.

\section{Resultados}

No Quadro 1 estão presentes os 10 artigos selecionados após busca orientada pela metodologia descrita nesta pesquisa. Os estudos foram organizados de acordo com os autores, ano, título do artigo, periódico, objetivos, e resultados da pesquisa, respectivamente. Os resultados da coleta foram organizados em três quadros baseados na combinação dos descritores: Infecções por Coronavírus (Coronavírus Infections) AND Pandemias (Pandemics) AND Saúde mental (Mental Health) AND Fisioterapia (Physiotherapy).O tema central dessa pesquisa foi encontrada em todos os estudos escolhidos. Os resultados referentes à caracterização das publicações deste estudo foram apresentados em 2 Quadros e 1 Tabela.

Quadro 1: Caracterização dos estudos selecionados por autores, ano, título do artigo, periódico, objetivos e resultados da publicação.

\begin{tabular}{|c|c|c|c|c|}
\hline $\begin{array}{c}\text { Autores/A } \\
\text { no }\end{array}$ & Título do artigo & Periódico & Objetivos & Principais resultados \\
\hline $\begin{array}{l}\text { Teixeira } \text { et } \\
\text { al. }(2020)\end{array}$ & $\begin{array}{l}\text { A saúde dos } \\
\text { profissionais de } \\
\text { saúde no } \\
\text { enfrentamento da } \\
\text { pandemia de } \\
\text { Covid-19. }\end{array}$ & $\begin{array}{c}\text { Ciênc. Saúde } \\
\text { Coletiva. }\end{array}$ & $\begin{array}{c}\text { Sistematizar um conjunto de } \\
\text { evidências científicas apresentadas em } \\
\text { artigos internacionais que identificam } \\
\text { os principais problemas que estão } \\
\text { afetando os profissionais de saúde } \\
\text { envolvidos diretamente no } \\
\text { enfrentamento da pandemia de } \\
\text { COVID-19 e apontam ações e } \\
\text { estratégias para a proteção e a } \\
\text { assistência à saúde desses } \\
\text { profissionais. }\end{array}$ & $\begin{array}{l}\text { Situando esta problemática na realidade brasileira, } \\
\text { este artigo resgata a análise dos problemas crônicos } \\
\text { que afetam os trabalhadores de saúde, decorrentes } \\
\text { do sub-financiamento do SUS, do congelamentos } \\
\text { dos gastos no setor, da deterioração dos serviços e } \\
\text { da precarização da força de trabalho e aponta os } \\
\text { desafios agudos que se apresentam à gestão do } \\
\text { trabalho e capacitação de pessoal, diante da } \\
\text { expansão da infraestrutura de leitos hospitalares e } \\
\text { da reorganização do processo de trabalho na } \\
\text { atenção básica para o enfrentamento da pandemia, } \\
\text { enfatizando as medidas necessárias para a proteção } \\
\text { e a promoção da saúde física e mental dos } \\
\text { profissionais e trabalhadores da saúde. }\end{array}$ \\
\hline $\begin{array}{c}\text { Fraga Maia } \\
\text { et al. } \\
(2020)\end{array}$ & $\begin{array}{c}\text { Fisioterapia } \\
\text { e COVID-19: das } \\
\text { repercussões } \\
\text { sistêmicas aos } \\
\text { desafios para } \\
\text { oferta de } \\
\text { reabilitação. }\end{array}$ & Edufba & $\begin{array}{c}\text { Discutir os impactos } \\
\text { funcionais decorrentes das } \\
\text { repercussões clínicas secundárias à } \\
\text { infecção pelo SARS-CoV-2, assim } \\
\text { como as abordagens e os desafios para } \\
\text { a oferta de atendimento } \\
\text { fisioterapêutico no âmbito do Sistema } \\
\text { Único de Saúde (SUS). }\end{array}$ & $\begin{array}{l}\text { É inegável que a pandemia de COVID-19 } \\
\text { modificou a forma como vivemos, cuidamos e } \\
\text { trabalhamos, e o processo de reabilitação deve se } \\
\text { adaptar a uma nova organização e gestão de } \\
\text { cuidado e assistência à saúde. }\end{array}$ \\
\hline
\end{tabular}




\begin{tabular}{|c|c|c|c|c|}
\hline $\begin{array}{l}\text { Oliveira et } \\
\text { al. }(2020)\end{array}$ & $\begin{array}{l}\text { COVID-19: do } \\
\text { enfrentamento ao } \\
\text { fortalecimento de } \\
\text { estratégias em } \\
\text { saúde mental - } \\
\text { uma revisão } \\
\text { narrativa. }\end{array}$ & Physis & $\begin{array}{l}\text { Verificar as informações veiculadas } \\
\text { por autoridades } \\
\text { sobre a saúde mental e a COVID-19. }\end{array}$ & $\begin{array}{l}\text { Nota-se a variedade de dispositivos de divulgação e } \\
\text { utilização de notas informativas aos profissionais e } \\
\text { gestores da saúde. Foram poucos os estados que se } \\
\text { preocuparam com rede psicossocial ativa, } \\
\text { especificamente no sentido de propor melhorias. }\end{array}$ \\
\hline $\begin{array}{c}\text { Cruz et al. } \\
(2020)\end{array}$ & $\begin{array}{c}\text { COVID-19 e } \\
\text { Saúde Mental: } \\
\text { Impacto na saúde } \\
\text { mental } \\
\text { relacionado à } \\
\text { pandemia de } \\
\text { COVID-19 nos } \\
\text { profissionais de } \\
\text { saúde do Brasil.. }\end{array}$ & $\begin{array}{l}\text { Forensic } \\
\text { Science } \\
\text { International: } \\
\text { Reports }\end{array}$ & $\begin{array}{c}\text { Avaliar o desenvolvimento de } \\
\text { transtornos mentais em coortes de } \\
\text { diferentes } \\
\text { profissionais de saúde (médicos, } \\
\text { enfermeiros, técnicos de enfermagem, } \\
\text { fisioterapeutas) } \\
\text { em relação ao grau de exposição e uso } \\
\text { de equipamento de proteção } \\
\text { individual (EPI), no período } \\
\text { epidêmico e pós-epidêmico; }\end{array}$ & $\begin{array}{l}\text { Os resultados do estudo poderão ter aplicabilidade } \\
\text { direta para promover o desenvolvimento de ações e } \\
\text { programas destinados ao cuidado em saúde dos } \\
\text { profissionais atuando na linha de frente em } \\
\text { pandemias. Além disso, através de um mapeamento } \\
\text { dos sintomas é possível identificar fatores de risco } \\
\text { e de proteção com a finalidade de reconhecer e } \\
\text { atuar na prevenção de desfechos psiquiátricos } \\
\text { anterior ao desenvolvimento de transtornos mentais } \\
\text { e suas consequências, bem como de desfechos } \\
\text { na saúde física que levam a incapacidade destes } \\
\text { profissionais para o trabalho. }\end{array}$ \\
\hline $\begin{array}{l}\text { Silva et al. } \\
\quad(2020)\end{array}$ & $\begin{array}{l}\text {. Efeitos da } \\
\text { pandemia no novo } \\
\text { Coronavírus na } \\
\text { saúde mental de } \\
\text { indivíduos e } \\
\text { coletividades }\end{array}$ & $\begin{array}{l}\text { J. Nurs. } \\
\text { Health. }\end{array}$ & $\begin{array}{l}\text { Desenvolver reflexões críticas sobre } \\
\text { os efeitos da pandemia do novo } \\
\text { Coronavírus na saúde mental de } \\
\text { indivíduos e coletividades. }\end{array}$ & $\begin{array}{l}\text { Tem-se percebido um pânico generalizado e } \\
\text { estresse na saúde mental na sociedade. Os achados } \\
\text { apontam um impacto psicológico imediato na } \\
\text { população em decorrência da pandemia, sendo } \\
\text { observado um crescimento de sintomas de } \\
\text { ansiedade, e até mesmo depressão. }\end{array}$ \\
\hline $\begin{array}{l}\text { Vasconcelo } \\
\text { s et al. } \\
(2020)\end{array}$ & $\begin{array}{l}\text { O novo } \\
\text { Coronavírus e os } \\
\text { impactos } \\
\text { psicológicos da } \\
\text { quarentena. } \\
\text { Desafios - } \\
\end{array}$ & $\begin{array}{c}\text { Revista } \\
\text { Interdisciplinar } \\
\text { da Universidade } \\
\text { Federal do } \\
\text { Tocantins }\end{array}$ & $\begin{array}{c}\text { Realizar uma análise sobre os efeitos } \\
\text { da quarentena e propor estratégias } \\
\text { para enfrentá-la, além de minimizar } \\
\text { seus efeitos }\end{array}$ & $\begin{array}{l}\text { Contudo, existem estratégias quando adotadas em } \\
\text { conjunto, podem minimizar os efeitos psicológicos } \\
\text { do isolamento e fazer desse momento menos } \\
\text { adoecedor. }\end{array}$ \\
\hline $\begin{array}{l}\text { Santana et } \\
\text { al. }(2020)\end{array}$ & & & $\begin{array}{l}\text { Compreender se o isolamento social } \\
\text { favorece o acometimento de } \\
\text { alterações psicológicas na população } \\
\text { mundial durante a pandemia de } \\
\text { COVID-19. }\end{array}$ & $\begin{array}{l}\text { As alterações psicológicas provocadas pelo } \\
\text { isolamento social mais apontadas foram: ansiedade, } \\
\text { depressão e estresse }\end{array}$ \\
\hline $\begin{array}{l}\text { Teixeira } e t \\
\text { al. }(2021)\end{array}$ & $\begin{array}{l}\text { A saúde dos } \\
\text { profissionais de } \\
\text { saúde no } \\
\text { enfrentamento da } \\
\text { pandemia de } \\
\text { Covid-19. Ciênc.. }\end{array}$ & Saúde Coletiva & $\begin{array}{l}\text { Rastrear sintomas de ansiedade, } \\
\text { depressão e estresse em } \\
\text { fisioterapeutas de um hospital público } \\
\text { de Goiás }\end{array}$ & $\begin{array}{l}\text { Houve maior prevalência de fisioterapeutas do sexo } \\
\text { feminino (73,5\%), o estresse obteve maior } \\
\text { prevalência entre os fisioterapeutas, com sintomas } \\
\text { de leve a extremamente severo em } 45 \% \text { deles, no } \\
\text { entanto, a ansiedade apresentou maior frequência } \\
\text { de sintomas moderados (15\%), seguidos por } \\
\text { extremamente severos (12\%) e graves (3\%). }\end{array}$ \\
\hline $\begin{array}{c}\text { Carvalho } \\
\text { (2020) }\end{array}$ & $\begin{array}{l}\text { O modo } \\
\text { psicossocial: Um } \\
\text { paradigma das } \\
\text { práticas } \\
\text { substitutivas ao } \\
\text { modo asilar. In } \\
\text { Paulo Amarante } \\
\text { (Org.), }\end{array}$ & $\begin{array}{l}\text { Ensaios: } \\
\text { Subjetividade, } \\
\text { saúde mental, } \\
\text { sociedade }\end{array}$ & $\begin{array}{l}\text { Avaliar o desenvolvimento de } \\
\text { transtornos mentais em coortes de } \\
\text { diferentes profissionais de saúde } \\
\text { (médicos, enfermeiros, técnicos de } \\
\text { enfermagem, fisioterapeutas) em } \\
\text { relação ao grau de exposição e uso de } \\
\text { equipamento de proteção individual } \\
\text { (EPI), no período epidêmico e pós- } \\
\text { epidêmico; }\end{array}$ & $\begin{array}{l}\text { Houve maior prevalência de sintomas de ansiedade, } \\
80 \% \text { dos residentes de fisioterapia apresentaram } \\
\text { sintomas grave e extremamente grave; quanto aos } \\
\text { sintomas estresse e depressão, } 50 \% \text { apresentaram } \\
\text { predomínio de gravidade leve; quanto ao nível de } \\
\text { atividade física, metade dos residentes de } \\
\text { fisioterapia são fisicamente inativos. }\end{array}$ \\
\hline $\begin{array}{l}\text { Negrini et } \\
\text { al,. (2020) }\end{array}$ & $\begin{array}{l}\text { Up to } 2.2 \text { million } \\
\text { people } \\
\text { experiencing } \\
\text { disability suffer } \\
\text { collateral damage } \\
\text { each day of } \\
\text { COVID19 } \\
\text { lockdown in } \\
\text { Europe. }\end{array}$ & $\begin{array}{l}\text { Eur J Phys } \\
\text { Rehabil Med. }\end{array}$ & $\begin{array}{l}\text { Avaliar a saúde mental, com foco na } \\
\text { ansiedade e depressão, de pacientes de } \\
\text { um ambulatório de fisioterapia frente } \\
\text { à pandemia da COVID-19. }\end{array}$ & $\begin{array}{l}\text { Portadores de doenças crônicas não transmissíveis } \\
\text { que estavam em reabilitação e tiveram seus } \\
\text { tratamentos suspensos por conta da pandemia da } \\
\text { COVID-19 apresentam escores da escala HADS } \\
\text { compatíveis com ansiedade e/ou depressão. }\end{array}$ \\
\hline $\begin{array}{l}\text { Pires } \\
(2021)\end{array}$ & $\begin{array}{c}\text { Identificar } \\
\text { sintomas de } \\
\text { ansiedade, } \\
\text { depressão e } \\
\text { estresse e o nível } \\
\text { de atividade física } \\
\text { em residentes de } \\
\text { fisioterapia de um } \\
\text { hospital público } \\
\text { de Goiás. }\end{array}$ & Saúde Coletiva & $\begin{array}{c}\text { O presente estudo apresentou maior } \\
\text { prevalência de sintomas de ansiedade } \\
\text { com maior frequência de sintomas de } \\
\text { maior gravidade, além disso metade } \\
\text { da amostra apresentou sintomas de } \\
\text { estresse e depressão, além disso } \\
\text { obteve um nível de atividade física } \\
\text { insatisfatório. }\end{array}$ & $\begin{array}{l}\text { Os fisioterapeutas lotados em um hospital público } \\
\text { apresentaram maior prevalência dos sintomas de } \\
\text { estresse e ansiedade, sendo que a depressão } \\
\text { apresentou a menor prevalência entre eles. } \\
\text { Servindo de alerta para os cuidados com a saúde } \\
\text { mental destes profissionais. }\end{array}$ \\
\hline
\end{tabular}

Fonte: Autores (2021). 
A Quadro 2 agrupa as variáveis país, ano, periódicos de publicação e abordagem metodológica. A maioria dos estudos localizados foram estrangeiros, totalizando 10 publicações, correspondendo a $100 \%$ das produções encontradas nas bases de dados virtuais, destacando-se a Brasil onde constatou-se mais produções sobre a temática com (09) artigos, correspondendo a $90 \%$ do total da amostra.

Pode-se observar que todas as publicações referentes ao tema foram em 2020 e 2021. Os periódicos que mais se destacaram foram Ciência e Saúde Coletiva com 30\%, revelando que os dados possuem cientificidade, impacto e seguem os princípios éticos na pesquisa.

à abordagem metodológica a que mais se destacou foi a revisão integrativa com $60 \%$ seguida da revisão sistemática com $20 \%$ pesquisa teórico-prática com $10 \%$ juntamente com ensaio científico.

Quadro 2: Distribuição das produções científicas segundo as variáveis: país, ano, periódicos, fator de impacto e delineamento do estudo. Teresina - PI, 2020.

\begin{tabular}{|c|c|c|}
\hline Variáveis & \multirow{2}{*}{$\mathbf{N}^{\mathbf{o}}$} & \multirow{2}{*}{$\%$} \\
\hline País & & \\
\hline Brasil & 09 & $90 \%$ \\
\hline Estados Unidos & 01 & $10 \%$ \\
\hline \multicolumn{3}{|l|}{ Ano } \\
\hline 2020 & 8 & $80 \%$ \\
\hline 2021 & 2 & $20 \%$ \\
\hline \multicolumn{3}{|l|}{ Periódico } \\
\hline Saúde Coletiva & 3 & $30 \%$ \\
\hline Edufba & 1 & $10 \%$ \\
\hline Physis & 1 & $10 \%$ \\
\hline Forensic Science International: Reports & 1 & $10 \%$ \\
\hline J. Nurs. Health. & 1 & $10 \%$ \\
\hline $\begin{array}{l}\text { Revista Interdisciplinar da } \\
\text { Universidade Federal do Tocantins }\end{array}$ & 1 & $10 \%$ \\
\hline $\begin{array}{l}\text { Ensaios: Subjetividade, saúde mental, } \\
\text { sociedade }\end{array}$ & 1 & $10 \%$ \\
\hline $\begin{array}{l}\text { Eur J Phys } \\
\text { Rehabil Med. }\end{array}$ & 1 & $10 \%$ \\
\hline
\end{tabular}

Fonte: Silva (2021).

O Quadro 3, representa os subtítulos utilizados na discussão dos artigos selecionados, para facilitar o entendimento e a discussão posterior do conteúdoespecificado. O foco do estudo, envolveu três subtemas: Consequências da pandemia COVID-19 na saúde mental dos profissionais de fisioterapia; Fatores associados às implicações na saúde mental dos fisioterapeutas, e; Estratégias para reduzir os riscos de agravos mentais. 
Quadro 3: Distribuição dos artigos em foco de estudo

\begin{tabular}{|c|c|}
\hline FOCO DO ESTUDO & $\begin{array}{c}\text { IDENTIFICAÇÃO DOS } \\
\text { ARTIGOS POR } \\
\text { AUTORES }\end{array}$ \\
\hline $\begin{array}{l}\text { Consequências da pandemia COVID-19 na saúde mental dos profissionais de fisioterapia } \\
\text {-Depressão } \\
\text {-Síndrome do pânico } \\
\text {-Sintomas de estresse } \\
\text { Redução da manifestação de emoções positivas e a perda de satisfação com a vida }\end{array}$ & $\begin{array}{c}\text { Teixeira } \text { et al. }(2020) \\
\text { Fraga Maia } \text { et al. }(2020) \\
\text { Oliveira } \text { et al. }(2020)\end{array}$ \\
\hline $\begin{array}{l}\text { Fatores associados às implicações na saúde mental dos fisioterapeutas } \\
\text {-Sentir-se vulnerável à COVID-19 por ser portador de DCNT, preocupação com a situação financeira e a insegurança } \\
\text { relacionada ao desencontro de informações chamaram a atenção como possíveis gatilhos para o desenvolvimento de episódios } \\
\text { ansiosos e/ou depressivos. } \\
\text { - Excesso de carga de trabalho } \\
\text { - Aumento do horário } \\
\text { - Grande quantidade de pacientes }\end{array}$ & $\begin{array}{c}\text { Cruz et al. (2020) } \\
\text { Silva et al. (2020) } \\
\text { Vasconcelos et al. }(2020)\end{array}$ \\
\hline $\begin{array}{l}\text { Estratégias para reduzir os riscos de agravos mentais } \\
\text {-A presença da atuação interdisciplinar é fundamental na abordagem dessas condições, pois a sobrecarga psicológica e de } \\
\text { trabalho dos profissionais ocasionada por esse momento crítico, pode tornar difícil a identificação dos casos de situação de risco } \\
\text { da saúde mental pelos profissionais especializados, assim, o fisioterapeuta deve abordar seu paciente de forma holística para } \\
\text { auxiliar na identificação e encaminhamento apropriado das situações de risco visando a saúde mental e o bem-estar geral. } \\
\text { - Diminuição da carga de trabalho } \\
\text { - Divisão dos horários e turnos }\end{array}$ & $\begin{array}{l}\text { Santana et al. }(2020) \\
\text { Abreu et al. }(2021) \\
\text { Carvalho (2020) } \\
\text { Pires (2021) }\end{array}$ \\
\hline
\end{tabular}

Fonte: BIBlioteca ViRTUAL Em SAÚde (BVS) (SCIELO, LILACS, BDENF, 2021).

\section{Discussão}

\subsection{Consequências da pandemia COVID-19 na saúde mental dos profissionais de fisioterapia}

Para Teixeira et al. (2020) cabe reiterar as recomendações da OMS com relação ao apoio que a população em geral pode dar aos profissionais e trabalhadores em saúde. Para os profissionais de saúde que estão na linha de frente do combate à pandemia, um estímulo necessário é o reconhecimento do esforço, até mesmo do sacrifício que muitos estão fazendo para continuar exercendo suas funções laborais nas condições em que trabalham. Saber que a família está segura, os amigos e a sociedade valorizam seu trabalho é fundamental para que eles consigam enfrentar com coragem e esperança a difícil tarefa em que estão empenhados. Considera-se a importância de instrumentalização dos gestores, trabalhadores e usuários do SUS, com o intuito de elaborar estratégias no enfrentamento à COVID-19.

Estudos realizados por Oliveira et al. (2020) enfatizam que o período de pandemia, em especial a quarentena, tende a aumentar de forma expressiva o sofrimento mental na população em geral, em decorrência do isolamento social e limitação da comunicação interpessoal. Além disso, os profissionais de saúde que estão na linha de frente possuem alto risco de desenvolver tais problemas, necessitando de intervenções que possam apoiá-los neste momento.

Cruz et al. (2020) corroboram que o impacto psicossocial estará presente nas diferentes fases da pandemia - inclusive após o seu fim, tornando-se necessário estruturar planos de ações amplos, que visem integrar estratégias de intervenções para a população em geral. Tais estratégias devem envolver as diferentes necessidades da população, de modo a alcançar as esferas sociais, culturais, religiosas e artísticas, as quais contribuem para a manutenção da saúde mental. Além disso, cada estado precisa avaliar as particularidades do seu território, de modo a desenvolver planos de cuidado em saúde mental que estejam em consonância com as recomendações da Organização Mundial de Saúde e do Ministério da Saúde.

Silva et al. (2020) afirmam que do ponto de vista da saúde mental, uma epidemia de grande magnitude implica em uma perturbação psicossocial que pode ultrapassar a capacidade de enfrentamento da população afetada. Pode-se considerar, inclusive, que toda a população sofre tensões e angústias em maior ou menor grau. Dessa forma é preciso que haja uma construção corresponsabilizada de enfrentamento entre os diversos atores sociais incluídos nesse processo, ou seja, a população, os dispositivos e autoridades sanitárias e o poder público. 


\subsection{Fatores associados às implicações na saúde mental dos fisioterapeutas}

Vasconcelos et al. (2020) Além dos problemas psicológicos surgidos em decorrência do isolamento social que foi imposto para a população mundial, bem como, as séries de restrições, incluindo, o fechamento de escolas, espaços culturais e de lazer, postos de trabalho, entre outros, bem como a imposição do chamando "lock down” , tudo isso contribuiu para o agravamento dos distúrbios psicológicos e o aparecimento de novos problemas psicológicos, inclusive, afetando os pacientes contaminados e que necessitaram de internação para o tratamento da doença. O papel deste profissional tornou-se indispensável e o enfrentamento da doença em seu conjunto - corpo e mente, destacando-se a necessidade de se remodelarem as atuações e formas de combate e busca de cura de diversos problemas psicológicos.

Contudo Santana et al. (2020) O COVID-19 já foi registrado em mais de 180 países ao redor do mundo e, com o avanço na contaminação da doença, diversos governantes adotaram diversas estratégias, com o intuito de retardar a progressão da doença (Kraemer et al. al. , 2020). Nesse contexto, o COVID-19 é transmitido de pessoa a pessoa, por gotículas de cuspo, tosse, catarro, acompanhados de contato pela boca, nariz ou olhos, ou mesmo por objeto se superfícies contaminadas. Atualmente, pesquisadores e profissionais de saúde enfrentam um desafio constante e à medida que aumenta o número de casos de COVID-19, pois a doença ainda não tem um risco clínico totalmente definido e o padrão de transmissibilidade, infectividade e letalidade. Dentre essas estratégias, a primeira medida é o distanciamento social, evitando áreas metropolitanas para manter as pessoas o pelo menos um metro e meio de distância, bem como proibindo eventos onde grande número de pessoas se reúna por exemplo.

De acordo com os estudos de Abreu et al. (2021), o paciente internado vê concretizada uma nova realidade a qual é incerta: não existe ainda protocolo de tratamento amplamente aceito. Por outro lado, o ambiente no qual ele é alocado a fim de ser tratado por vezes é um ambiente novo, feito sem a mesma estrutura que um hospital convencional. É indiscutível que a pandemia do novo coronavírus (COVID-19), bem como as notícias e atos governamentais dela decorrentes ocasionaram emoções, receios, e sentimentos conflitantes em muitas pessoas. Os mais fragilizados para esta situação são exatamente os que estão internadas, infectadas pelo novo vírus, e esta nova demanda deve ter acompanhamento do profissional em psicologia, que irá se valer, especificamente do ramo denominado Psicologia Hospitalar e da Saúde.

Pires (2021) os pacientes os Cuidados diferenciados e humanizados em pacientes graves acometidos pela COVID 19 em UTI requerem monitoramento regular dos sinais vitais e, sempre que possível, utilização de escores de alerta que facilitam o reconhecimento precoce e a intensificação do tratamento do paciente em deterioração.

Os exames laboratoriais de hematologia e bioquímica, gasometria, raio X de tórax e o ECG devem ser realizados na admissão e conforme indicação clínica para monitorar as complicações, como lesão hepática aguda, lesão renal aguda, lesão cardíaca aguda, piora da oxigenação ou instabilidade hemodinâmica. Exames de coagulação, como D-dímero, TP e TPPA, devem ser solicitados nos casos mais graves, bem como ecocardiograma e tomografia de tórax (quando disponíveis e possíveis).

Em casos suspeitos ou confirmados para SARS-CoV-2 que não necessitem de hospitalização e o serviço de saúde opte pelo isolamento domiciliar, o médico poderá solicitar raios-X de tórax, hemograma e provas bioquímicas antes de serem dispensados para o domicílio, conforme avaliação clínica do paciente. Esses pacientes deverão receber orientações de controle de infecção, prevenção de transmissão para contatos e sinais de alerta para possíveis complicações. Um acesso por meio de comunicação rápida deve ser providenciado para eventuais dúvidas ou comunicados.

A presença de qualquer sinal de alerta deverá determinar retorno e hospitalização imediata do paciente. Porém, é necessário avaliação individualizada do caso, considerando também se o paciente apresenta condições ou fatores de risco e se o ambiente residencial é adequado, e se o paciente é capaz de seguir as medidas de precaução recomendadas pela equipe de saúde responsável pelo atendimento 


\subsection{Estratégias para reduzir os riscos de agravos mentais}

Algumas estratégias para reduzir os riscos de agravos mentais de acordo com Santana et al. (2020) refere-se ao contingenciamento de procedimentos eletivos de hospitais deverá ser considerado de acordo com o momento epidemiológico local, especialmente, quando identificada a transmissão comunitária. No Brasil esse contingenciamento deverá ser particularizado e regionalizado, devido à expectativa de a pandemia ocorrer em diferentes momentos de pico no país. Recomenda-se aos serviços de saúde o estabelecimento de fluxo diferenciado, com áreas exclusivas para o atendimento de pacientes sintomáticos respiratórios e a implementação de coortes de pacientes entubados pela COVID-19, na ausência/ impossibilidade/escassez de áreas de isolamento. Além disso, indica-se que seja disponibilizada ala completa para atendimento aos pacientes com a COVID-19 para evitar infecção cruzada e possibilitar racionalização de recursos e profissionais.

Abreu et al. (2021) Tais problemas devem ser analisados e superados da melhor forma pelo psicólogo hospitalar e da saúde, sempre tendo em vista a melhor orientação para o paciente. Ainda, há que se somar que, por se tratar de uma doença contagiosa, estes profissionais também enfrentam o perigo de contágio com a doença, ou seja, para o enfrentamento da pandemia exigiu-se novas formas de atuação deste profissional.

Uma das grandes aliadas do processo de estabilização segundo Carvalho (2020) emocional, no início da fase de resposta a uma pandemia, é a confiança estabelecida em protocolos e orientações das autoridades sanitárias à população em geral e aos trabalhadores da linha de frente. Essa relação potencializa a sensação de ser cuidado e, ao mesmo tempo, evoca o sentimento de altruísmo ao cuidar dos outros na medida em que se evita sair de casa.

Pires (2021) acrescenta que estabelecer uma rotina diária para si e para a família, incluindo rotina de alimentação e sono. O planejamento auxilia no enfrentamento do estresse, uma vez que aciona a sensação de retomada de controle e favorece a tomada de decisões de maneira consciente. Uma rotina estabelecida de maneira intencional, com descrição das tarefas, assim como da sua duração, possui maior efeito do que aspirações gerais sobre o que deve ser feito. O planejamento da rotina é essencial, sobretudo em famílias com crianças, na medida em que proporciona certa previsibilidade nas atividades cotidianas.

\section{Considerações Finais}

A pandemia de Covid-19, está tendo um enorme impacto piscicológico nos indivíduos. A maioria dos indivíduos sofria de distúrbios moderados, enquanto os sujeitos que relatam severos sintomas eram uma minoria. Algumas categorias se mostraram mais vulneráveis, ou seja, profissionais de saúde e pacientes afetados pelo CoViD-19. Nos observados a prevalência não era homogênea, sexo feminino e idade mais jovem foram associados com maior risco para os resultados da saúde mental.

Ainda pouco se conhece sobre as consequências emocionais e mentais em profissionais de saúde diretamente expostos à COVID e a relação destas consequências com as diferentes categorias de riscos relacionados às condições laborais e níveis de exposição. Esse conhecimento será necessário para um planejamento de saúde amplo e que considere medidas de prevenção a médio e a longo prazo no âmbito do desempenho profissional.

Os efeitos da pandemia vêm atingindo direta e indiretamente a saúde mental das pessoas nos mais diversos aspectos, o que implica numa condição preocupante de saúde pública.

A quarentena pode desencadear sintomas psicológicos, sendo os sintomas mais comuns ansiedade, tristeza e raiva, podendo ser pontuais ou se estenderem após o término do isolamento.

Infere-se que o isolamento social durante a pandemia do COVID-19 interfere na qualidade de vida das pessoas, e contribui para o comprometimento da saúde mental.

Diante de tudo que foi exposto pelos autores pode-se dizer que todos os profissionais tanto fisioterapuetas quando de maneira geral que estavam e ainda estão na linha de frente precisam de ajuda ao que diz respeito a saúde mental. 


\section{Referências}

Amarante, PDC. (2203). A (clínica) e a reforma psiquiátrica. In: Amarante PDC (org). Arquivos de saúde mental e Atenção Psicossocial, Nau;

Basaglia, Franco. (2007). A psiquiatria alternativa: contra o pessimismo da razão o otimismo da prática. Conferências no Brasil. São Paulo: Mosanto, institucional. Cortez.

Brasil. Ministério da Saúde. Centro de Documentação. (2016). Primeira Conferência Nacional de Saúde Mental. Brasília, DF. Relatório final.

Brasil. Ministério da Saúde. Portaria n ${ }^{\text {189, de }} 11$ de dezembro de 1991. Dispõe sobre o atendimento à Saúde Mental no Brasil. Diário Oficial Da República Federativa do Brasil. Brasília, DF, 11 dez. 2012. Seção 1, p. 28495.

Campos, S., Barros, M. Castro, 1. (2015). Saúde Mental em Saúde Pública: um percurso histórico, conceitual e as contribuições da psicologia nesse contexto. Psicólogo Informação, São Paulo, n. 6/7.

Carvalho, Abílio. (2020). O modo psicossocial: Um paradigma das práticas substitutivas ao modo asilar. In Paulo Amarante (Org.), Ensaios: Subjetividade, saúde mental, sociedade (pp. 141-168). Fiocruz.

Carvalho, M. L. R. (2001). A dinâmica da razão na filosofia de Espinosa. Lisboa: Fund. Calouste Gulbenkian/Junta. Nac. de Inv. Cient. e Tec.

CFO. Conselho Federal de Odontologia. (2020). Manual de boas práticas em biossegurança para ambientes odontológicos. Rio de Janeiro,

Chinese Sars Molecular Epidemiology Consortium et al. (2004).Molecular Evolution of the SARS coronavirus during the course of the SARS epidemic in China. Science, 303(5664), 1666-1669,

Costa Neto, M.M., Silvestre, J.A. (2009). Atenção à Saúde do Idoso - Instabilidade Postural e Queda. Caderno de Atenção Básica voltado para a atenção à pessoa idosa. Brasília: Departamento de Atenção Básica, Secretaria de Políticas de fisioterapia, Ministério da Saúde,

Costa-Rosa, A. (2010). Saúde Mental Comunitária: Análise dialética de um movimento alternativo. Dissertação de mestrado não publicada, Instituto de Psicologia, USP, São Paulo.

Cruz et al,. COVID-19 e Saúde Mental: Impacto na saúde mental relacionado à pandemia de COVID-19 nos profissionais de saúde do Brasil. (2020). Forensic Science International: Reports. v. 2, 2020.

Dalbello, M. S. B., Araújo, F. (2016). A reforma e os hospitais psiquiátricos: histórias da desinstitucionalização. Psicologia \& Sociedade, 22(1), 112-120,

Delgado, S. (2009). Reforma Sanitária Brasileira: dilemas entre o instituinte e o instituído. Ciência \& Saúde Coletiva, Rio de Janeiro, 14(3), 743-752,

Dimenstein, F. (2015). A reforma psiquiátrica brasileira, da década de 1980 aos dias atuais: história e conceito. História, Ciências, Saúde Manguinhos, Rio de Janeiro $9(1), 25-59$.

Fraga-Maia, H., Pinto, E. B., Aleluia, Í. R. S., Cavalcante, L. L. R., Pedreira, R. B. S., Silva, T. De J., Souza, T. S. De, Pinto, J. M., Pinto Junior, E. P. (2020). Fisioterapia e COVID-19: das repercussões sistêmicas aos desafios para oferta de reabilitação. In: BARRAL-NETTO, M.; Barreto, M. L.; Pinto Junior, E. P.; Aragão, E. (org.). Construção de conhecimento no curso da pandemia de COVID-19: aspectos biomédicos, clínico-assistenciais, epidemiológicos e sociais. Salvador: Edufba, v. 1.

Franco, Aline Batista Gonçalves et al. (2020).Atendimento odontológico em UTI's na presença de COVID-19. InterAmerican Journal of Medicine and Health, 3, e20200304-e20200304.

Franco, Aline Batista Gonçalves et al. (2020). Atendimento odontológico em UTI's na presença de COVID-19. InterAmerican Journal of Medicine and Health, v. 3, p. e20200304-e20200304,

Gladys, E. F.; Swain, M. (2016). Integralidade e construção de novas profissionalidades no cotidiano dos serviços substitutivos de saúde mental. In: Revista de Terapia Ocupacional, 17(3), set-dez,

Jb, Franco, De Camargo, A. R., Mpsm, Peres. (2020).Cuidados odontológicos na era do COVID-19: recomendações para procedimentos odontológicos e profissionais. Rev Assoc Paul Cir Dent, 74(3), 18-21.

Kochhar, Anuraj Singh et al. (2020).Provision of Continuous Dental Care for Oral Oncology Patients during \& after COVID-19 Pandemic. Oral Oncology.

Mangia, A. T. M., Muramoto, A. A. L. (2016). Problematizando a reforma psiquiátrica brasileira: a genealogia da reabilitação psicossocial. Psicologia em Estudo, Maringá, 15(1), 27-34.

Meng, L., Hua, F., Bian, Z. Coronavirus disease. (2019). COVID-19: emerging and future challenges for dental and oral medicine. Journal of Dental Research, 99(5), 481-487,

Negrini S, Grabljevec K, Boldrini P, Kiekens C, Moslavac S, Zampolini M, Et Al. 2020. Up to 2.2 million people experiencing disability suffer collatera damage each day of COVID19 lockdown in Europe. Eur J Phys Rehabil Med.

Oliveira et al,. 2020. COVID-19: do enfrentamento ao fortalecimento de estratégias em saúde mental - uma revisão narrativa. Physis, Rio de Janeiro, 29(2), e290213.

Pedrosa, B. (2014). Libertando identidades: da reabilitação psicossocial à cidadania possível. Rio de Janeiro: Te Corá/Instituto Franco Basaglia,.

PIRES, Lorrane Gabriela Lessa. (2021). Saúde mental e nível de atividade física de residentes de fisioterapia durante a pandemia de COVID-19. Revista Pontifícia Universidade Católica de Goiás. 
Ribeiro, S., Martins, P., Oliveira, G., Lima, L. B. (2015). Avaliação das limitações no comportamento social em pacientes psiquiátricos de longa permanência. Ciência \& Saúde Coletiva, Rio de Janeiro, 06(1), 105-113.

Saraceno, B. (2015). Libertando identidades: da reabilitação psicossocial à cidadania possível. Rio de Janeiro: Te Corá/Instituto Franco Basaglia.

Sexton J, Wilson A, Sassi H, Et Al. (2020). Rastrear e controlar a contaminação de superfícies macias em ambientes de fisioterapia. Am J Infect Control.

Silva Hgn, Santos Les, Oliveira Aks. (2020). Efeitos da pandemia no novo Coronavírus na saúde mental de indivíduos e coletividades. J. Nurs. Health. 10(n.esp.):e20104007

Silveira, L., \& Corrêa, K. (2021). Rastreio de sintomas de depressão, ansiedade e estresse em fisioterapeutas durante a pandemia do Covid 19. Movimenta (ISSN 1984-4298), 14(1), 107-119.

Teixeira et al,. (2020). A saúde dos profissionais de saúde no enfrentamento da pandemia de Covid-19. Ciênc. Saúde Coletiva. Set.

Vasconcelos, C. S. S., Feitosa, I. O., Medrado, P. L. R., Brito, A. P. B. (2020). O novo Coronavírus e os impactos psicológicos da quarentena. Desafios Revista Interdisciplinar da Universidade Federal do Tocantins, 7(3), 75-80.

Vidal, M. B. S.,Bandeira, S. T. F.,Gontijo, L. R. (2015). Familiares de usuários vivenciando a transformação do modelo assistencial psiquiátrico. Estudos de Psicologia, 14(2), 133-140. 